\title{
TTR
}

Traduction, terminologie, re?daction

\section{Les formations en traduction et interprétation : Perspectives en Europe de l'Ouest}

\section{Monique Caminade}

Volume 8, numéro 1, 1er semestre 1995

Orientations européennes en traductologie

URI : https://id.erudit.org/iderudit/037204ar

DOI : https://doi.org/10.7202/037204ar

Aller au sommaire du numéro

Éditeur(s)

Association canadienne de traductologie

ISSN

0835-8443 (imprimé)

1708-2188 (numérique)

Découvrir la revue

Citer cet article

Caminade, M. (1995). Les formations en traduction et interprétation :

Perspectives en Europe de l'Ouest. TTR, 8(1), 247-270.

https://doi.org/10.7202/037204ar
Résumé de l'article

Les formations en traduction et interprétation : Perspectives en Europe de l'Ouest - Cet article propose une analyse globale des programmes de formation et interprétation dans les pays d'Europe de l'Ouest. L'analyse s'effectue autour de trois axes 1) le statut de ces formations dans le cadre institutionnel de l'enseignement supérieur des différents pays; 2) le profil des formations (courtes, moyennes, longues); 3 ) le rapport des disciplines de la traduction et de l'interprétation au sein de ces formations. Cette analyse a été faite sur la base d'un recensement de 136 institutions dans dix-sept pays au cours de l'année 1994.
Tous droits réservés @ C TTR: traduction, terminologie, rédaction — Les auteurs, 1995
Cedocument est protégé par la loi sur le droit d'auteur. L'utilisation des services d'Érudit (y compris la reproduction) est assujettie à sa politique d'utilisation que vous pouvez consulter en ligne.

https://apropos.erudit.org/fr/usagers/politique-dutilisation/ 


\section{Les formations en traduction et interprétation: Perspectives en Europe de l'Ouest}

\section{Monique Caminade}

\section{Introduction}

La nécessité d'une information globale sur la formation des traducteurs et interprètes n'est plus à mettre en doute. Si elle est très fortement ressentie par les institutions elles-mêmes, elle se justifie surtout par la volonté de changer un peu la réflexion sur cette formation. La plupart des publications et communications sur le sujet se résument à des descriptions particulières de curriculae ou à des directives sur la façon dont devrait généralement s'organiser cette formation. On assiste de part et d'autre à des tentatives de normalisation autour de certains modèles ou de certains discours théoriques qui tendent trop souvent à ignorer la diversité des formations existantes. Il manque donc à toutes ces initiatives une étude globale, un cadre de discussion reposant sur des données réelles, qui viendrait à faire de la formation des traducteurs et interprètes non plus un ensemble de projets épars mais un domaine cohérent de réflexion et de débat.

Ce manque d'information globale nous a conduite, au cours de l'année 1994, à faire un recensement des formations en traduction 
et interprétation qui constitue la source principale de cet article' 1 La démarche suivie pour ce travail se situe à l'opposé des démarches qui tendraient à définir a priori certains critères de qualité et à établir ensuite une sélection des formations - ou programmes d'études. Il nous a semblé que la démarche normative ne permettait pas de faire apparaître la réelle diversité des formations. Nous avons alors choisi un critère suffisamment large pour pouvoir embrasser les multiples aspects de la traduction et de l'interprétation dans le cadre des formations de l'enseignement supérieur: nous avons relevé dans chaque pays les programmes d'études débouchant sur un diplôme - ou titre - portant la mention de «traducteur», «interprète», «traduction», «interprétation» ou tout terme dérivé: «traductique», «traductologie», etc. Ce critère peut-être discuté - la sélection ou non-sélection de certaines formations n'est d'ailleurs pas sans poser problèmes - mais il a l'avantage de garantir une certaine objectivité. Sur 136 institutions recensées en Europe de l'Ouest, nous avons relevé 218 diplômes à tous les niveaux de formation au sein de l'enseignement supérieur.

Une telle entreprise est d'autant plus pressante aujourd'hui que la formation des traducteurs et interprètes a pris un véritable essor depuis le milieu des années 80 . Le schéma suivant montre l'évolution du nombre d'institutions concernées et non des programmes d'études. Mais il donne une indication assez précise de l'importance croissante des disciplines de la traduction et de l'interprétation dans l'enseignement supérieur en l'Europe de l'Ouest.

1. Monique Caminade et Anthony Pym, Annuaire mondial des formations en traduction et interprétation, numéro spécial de la revue Traduire (Société française des Traducteurs, Paris) qui devrait paraitre en septembre 1995. Le présent article a été rédigé sur la base d'un premier recensement qui concernait uniquement les pays d'Europe de l'Ouest (Allemagne, Autriche, Belgique, Danemark, Espagne, Finlande, France, Grande-Bretagne, Grèce, Irlande, Italie, Luxembourg, Norvège, Pays-bas, Portugal, Suède, Suisse). 
Les formations en traduction et interprétation se sont développées en Europe de l'Ouest à partir des années 40-50 dans le cadre d'écoles supérieures ou d'instituts privés. Ces écoles ou instituts ont été ensuite progressivement intégrés au domaine univer-

\section{Fréquence de création des formations de traducteurs et d'interprètes} (nombre d'institutions sur intervalles de 3 ans)

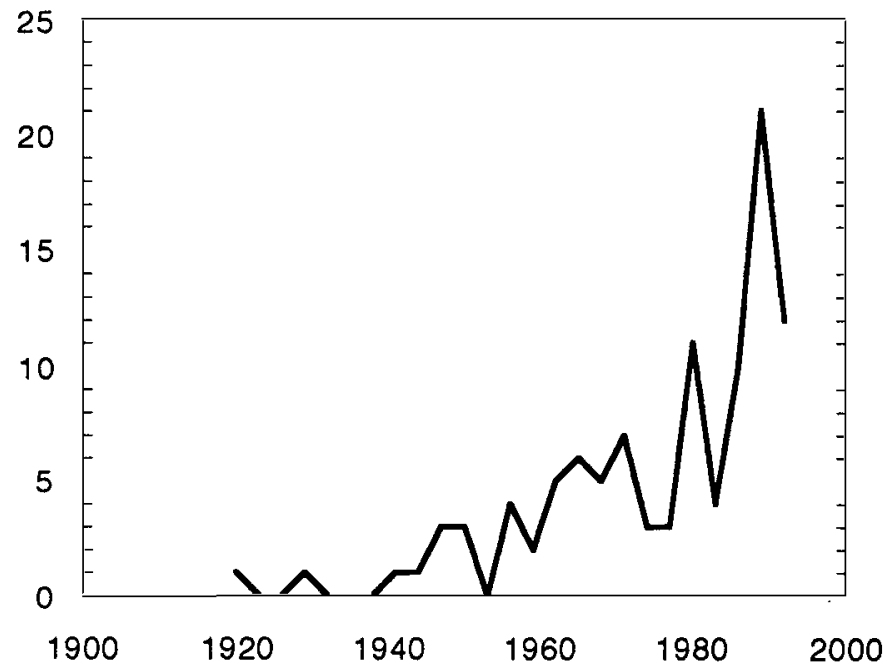

sitaire. Les formations en traduction et interprétation qui ont commencé dans les années 60 n'ont pas échappé non plus à ce processus d'intégration. Si les formations étaient relativement peu nombreuses au départ, elles ont progressé de façon constante mais assez lente jusqu'au début des années 80 . On observe ensuite, à partir des années 83-85, une évolution beaucoup plus rapide: entre les années 85 et 90 , plus d'une vingtaine de formations ont vu le jour. Ce fait s'explique d'une part par l'introduction des réformes dans ces années-là dans les divers systèmes universitaires et d'autre part par le fait que la traduction est perçue comme une profession d'avenir. Il semble qu'aujourd'hui cet enthousiasme ait un peu diminué. La difficulté à définir un objet scientifique concernant la traduction et le manque de reconnaissance persistant des professions de la traduction au niveau de la société, ainsi sans doute que la récession économique, introduisent un doute sur la pertinence de 
tous ces projets, d'où le ralentissement des créations, tout au moins en Europe de l'Ouest, à partir des années 90 .

\section{Une triple diversité}

À cette évolution irrégulière s'ajoute un autre constat: l'extrême diversité des formations sur l'ensemble des pays qui nous concernent. Diversité qui donne à première vue le sentiment que les disciplines de la traduction et de l'interprétation n'ont pas encore trouvé leur place dans le cadre de l'enseignement supérieur. Cette diversité se manifeste à trois égards au moins. Tout d'abord, le statut des formations en traduction et interprétation dans l'enseignement supérieur. $\mathrm{Si}$, dans certains cas, les formations demeurent purement professionnelles, s'inscrivent dans des structures séparées (écoles ou instituts) et débouchent sur des types de diplôme qui n'entrent pas dans le cadre universitaire, elles sont, dans d'autres cas, tout à fait assimilées aux formations universitaires traditionnelles et débouchent parfois sur des formations doctorales. Cela veut dire que la conception de la traduction et de l'interprétation et la place qu'on leur réserve dans l'enseignement supérieur diffèrent considérablement d'un pays à l'autre, voire à l'intérieur des pays eux-mêmes.

Ensuite, le profil des formations - nombre d'années et niveau d'études - au sein de l'enseignement supérieur varie aussi considérablement. Les études en traduction et interprétation peuvent en effet se réaliser à travers trois types de formation: les formations longues et moyennes (respectivement de quatre ou cinq ans et de trois ans, à partir de l'accès à l'enseignement supérieur), ainsi que les formations courtes, de moyen ou haut niveau (un an après une formation universitaire de trois ou quatre ans). Cet aspect revêt une certaine importance dans le discours théorique sur la formation des traducteurs et interprètes. On a parfois tendance à utiliser le profil des formations comme critère de qualité en particulier en ce qui concerne l'interprétation de conférence. Les bonnes formations professionnelles seraient essentiellement, selon certains, des formations courtes de haut niveau. Or, le profil des formations s'explique le plus souvent par la différence des systèmes éducatifs 
nationaux. L'Allemagne, par exemple, présente des cursus universitaires compacts. Le Diplom se prépare en quatre ans sans diplôme intermédiaire et les formations post-universitaires en traduction sont toutes des formations doctorales. La France, en revanche, présente un système universitaire beaucoup plus éclaté. Les études universitaires se divisent en trois cycles et chaque cycle débouche sur un diplôme propre; le deuxième cycle comprend deux diplômes: la licence, puis la maîtrise qui donne accès au troisième cycle; le troisième cycle comprend soit des formations professionnelles courtes (diplôme d'études supérieures spécialisées, DESS) soit des formations doctorales. Le système français favorise donc mieux qu'en Allemagne le développement des formations professionnelles courtes de haut niveau. Mais cela ne veut pas dire pour autant que l'Allemagne n'offre aucune bonne formation professionnelle en traduction ou en interprétation.

On trouve enfin un autre élément de diversité dans le rapport que les formations établissent entre les deux disciplines de la traduction et de l'interprétation (voir aussi section 4). Parfois, ces deux disciplines font l'objet d'un cursus global de formation. Parfois, elles sont enseignées à travers des cursus tout à fait séparés. $\mathrm{Ou}$ encore, elles s'inscrivent dans des cursus combinés: une formation commune débouchant sur deux cursus spécialisés (traduction ou interprétation) ou deux formations successives, en traduction d'abord, en interprétation ensuite. Le rapport traduction/ interprétation nous paraît fondamental en raison des conséquences qui pourraient en découler pour les formations dans le futur. On observe en effet une sorte de rivalité entre deux modèles opposés: le modèle qui tend à faire de la traduction et de l'interprétation deux disciplines séparées qui correspondraient à deux activités professionnelles distinctes et le modèle où ces deux disciplines sont enseignées globalement, considérées alors dans le cadre général des professions de la communication interculturelle. On ne forme plus des traducteurs ou des interprètes, mais des professionnels de la communication interculturelle qui seront à même de s'adapter aux multiples visages de la traduction. 
L'idée qu'il y aurait un modèle européen de formation en traduction et interprétation ne s'appuie donc sur aucune réalité. Nous ferons une sorte «d'état des lieux» des formations en Europe de l'Ouest autour de ces trois points de diversité. En ce qui concerne le premier point, nous ferons une brève description du statut des formations dans l'enseignement supérieur de chaque pays. Cette façon de procéder - pays par pays - est peut-être un peu lourde, mais il y a trop peu de ressemblance d'un pays à l'autre et trop de particularités au sein des pays eux-mêmes pour envisager une description plus synthétique. Il n'en est pas ainsi, en revanche, pour les deux autres points. Comme nous venons de le voir, on peut déceler, tant du point de vue du profil des formations que du rapport traduction/interprétation certains modèles qui se retrouvent d'un pays à l'autre et dont l'étude nous permettra de mettre en évidence quelques tendances générales de la formation des traducteurs et interprètes.

\section{Le statut des formations}

S'il est vrai que la distinction entre formations universitaires et non universitaires tend à perdre de sa pertinence, elle nous permet tout de même, en ce qui concerne la traduction et l'interprétation, d'obtenir quelques indications sur la façon dont ces disciplines sont aujourd'hui perçues dans le cadre de la formation. En effet, les formations de traducteurs et interprètes étaient au départ, c'est-à-dire dans les années 40-60, essentiellement axées sur la pratique. Beaucoup furent créées pour former des professionnels destinés à travailler dans des domaines très spécifiques (armée, ministères de l'économie et des finances, des affaires étrangères, grandes organisations internationales, etc.). Elles s'inscrivaient de ce fait essentiellement - si ce n'est exclusivement - dans le cadre de structures (écoles ou instituts) indépendantes du cadre universitaire traditionnel.

Aujourd'hui, la généralisation des professions de la traduction et de l'interprétation dans une société de plus en plus multiculturelle et les interrogations théoriques dont elles font l'objet tendent à inscrire ces disciplines dans un domaine d'études et de 
recherches propre (traductologie, translation studies, Übersetzungswissenschaft, etc.). Ceci justifie la création de cursus à la fois plus généraux et plus diversifiés du point de vue des débouchés et plus scientifiques du point de vue des contenus. L'enseignement des théories de la traduction prend une place de plus en plus importante dans le cadre des formations courantes et l'on voit se développer, parallèlement, des formations doctorales en traductologie. Les cursus en traduction et interprétation acquièrent ainsi un profil de plus en plus identifiable à celui des formations universitaires traditionnelles.

C'est peut-être ce qui explique qu'à l'heure actuelle globalement $70 \%$ environ des formations en traduction et interprétation s'inscrivent dans le cadre des formations universitaires ou rattachées à l'université et $30 \%$ seulement dans le cadre des formations non universitaires. Ces proportions varient bien sûr d'un pays à l'autre.

Les formations universitaires en traduction et interprétation prennent place de deux manières au sein de l'université: a) elles bénéficient de structures propres (départements, facultés ou écoles internes consacrés à la traduction et à l'interprétation); b) elles s'intègrent à des structures relevant d'autres disciplines (essentiellement langues, linguistique, langues et linguistique appliquées, littérature et éventuellement économie et commerce). Voici un relevé substantiel, non exhaustif, pays par pays, de ces formations.

Allemagne: En dehors de deux Fachhochshulen (Cologne et Flensburg) offrant des diplômes professionnels de trois et quatre ans et de deux institutions non universitaires (Munich et Würzburg), les formations en traduction et en interprétation sont toutes parties prenantes de l'Université et débouchent sur des Diplom de quatre ans. Certaines appartiennent à des départements ou des facultés de langues, de langues appliquées ou de littérature (par exemple Berlin, Germersheim, Düsseldorf). D'autres font l'objet de structures propres au sein d'une université, d'une faculté ou d'un département. C'est le cas, par exemple, de l'Institut für Übersetzen und 
Dolmetschen de l'Université de Heidelberg, de l'Institut für Fremdsprachenvermittlung intégré au département de linguistique appliquée de l'Université de Halle, ou de l'Institut für Sprach-und Übersetzungswissenschaft de l'Université de Leipzig.

Autriche:Les formations autrichienness'apparentent aux formations allemandes de Heidelberg, Halle ou Leipzig. Les instituts universitaires de traducteurs et d'interprètes par exemple à Innsbruck et à Vienne délivrent des diplômes équivalents au Diplom allemand.

Belgique: La formation des traducteurs et interprètes en Belgique relève principalement de l'enseignement supérieur non universitaire de type long. Seules les formations de Mons et d'Anvers sont administrativement rattachées à des universités. L'Université de Liège offre aussi une maitrise en traduction dans le cadre du Département de Langues et Littératures germaniques. La nouvelle réforme universitaire actuellement à l'œuvre dans tout le pays préconise un rattachement des formations non universitaires à l'université. Mais les résistances rencontrées au sein même des universités remettent en cause, pour l'instant, ce processus. Ceci dit, les cursus non universitaires de type long (4 ans) - où se situe la formation des traducteurs et interprètes - présentent le même profil et aboutissent aux mêmes diplômes de licencié traducteur ou licencié interprète que dans le cadre universitaire. Il est à remarquer qu'en Belgique, en dehors de l'Institut de Linguistique de Mons et du département de Langues et Littératures germaniques de l'Université de Liège, toutes les formations universitaires et non universitaires ont leurs structures propres (départements ou instituts de traducteurs et d'interprètes).

Danemark: Le cas du Danemark est particulier. Les seules formations qui délivrent des diplômes professionnels en traduction et interprétation sont les deux écoles de commerce d'Aarhus et de Copenhague. Ces établissements jouissent d'un statut particulier au sein de l'enseignement supérieur dans la mesure où ils sont privés. Mais les diplômes délivrés sont universitaires. L'école de commerce de Copenhague comprend une faculté des langues modernes dans laquelle on trouve un double cursus de formation en langages 
spécialisés d'une part et en interprétation de conférence d'autre part. La traduction et l'interprétation sont donc intimement liées, au Danemark, aux formations commerciales. Il s'agit en gros de former des professionnels hautement qualifiés dans le domaine de la communication interlinguistique pour les besoins très précis de l'industrie et du commerce. L'Université de Copenhague abrite néanmoins aussi un Centre d'études en traduction et en lexicographie qui forme par exemple des traducteurs spécialisés en sous-tirage.

Espagne: Toutes les formations en traduction et interprétation en Espagne sont des formations universitaires longues (licenciaturas). Ceci est le fait d'une réforme récente. Jusqu'en 1992, l'enseignement de la traduction et de l'interprétation n'était représenté - en dehors de quelques formations telles que celle de l'Université Complutense de Madrid - que par trois écoles universitaires délivrant des diplomas ( 3 années d'études): 1'Université Autonome de Barcelone, l'Université de Grenade et l'Université de Las Palmas de Gran Canaria. Entre 1992 et 1994, six facultés de traduction et d'interprétation se sont créées et 12 licenciaturas en traducción e interpretación ont vu le jour, soit dans le cadre de ces facultés, soit dans le cadre de facultés ou de départements de langues. L'Espagne est le seul pays, en Europe de l'Ouest, qui présente des facultés universitaires en traduction et interprétation.

Finlande: Toutes les formations finlandaises en traduction et interprétation sont universitaires et bénéficient de structures propres; il y a quatre départements ou écoles de ce niveau. Elles aboutissent à des diplômes universitaires, après 5 ou 6 ans d'études. L'interprétation de conférences fait l'objet, depuis peu, d'une formation nationale, intensive, à Turku.

France: La grande majorité des formations en traduction et interprétation en France sont des formations universitaires courantes aboutissant à des maitrises de Langues étrangères appliquées mention traduction spécialisée (deuxième cycle) ou à des diplômes d'études supérieures spécialisées (DESS) de traduction ou 
d'interprétation (troisième cycle). Elles s'inscrivent pour la plupart dans des départements de langues, littératures et civilisations étrangères ou de langues étrangères appliquées. Les structures propres, exclusivement consacrées à l'enseignement de la traduction ou de l'interprétation, sont des écoles ou instituts qui peuvent être intégrés soit à une université, par exemple l'École Supérieure d'Interprètes et de Traducteurs (ESIT), soit à l'enseignement supérieur technique privé, cas de l'Institut Supérieur d'Interprétation et de Traduction (ISIT). Ces deux institutions ont été créées en 1957. L'ESIT fut intégrée en 1984 à l'Université de Paris 3 avec un statut d'école interne. L'ISIT, qui dépend de l'Institut catholique de Paris ${ }^{2}$, fut homologuée par le Rectorat de l'Académie de Paris comme établissement d'enseignement supérieur technique privé en 1992. L'ESIT offre un double DESS de traduction et d'interprétation de deux ans à partir de la Licence (le DESS se prépare habituellement en un an après la Maîtrise). Le Titre post-universitaire d'interprète de conférence de l'ISIT s'organise de la même façon. D'autres institutions consacrées à la traduction et à l'interprétation existent: elles sont situées à Angers, Toulouse, Strasbourg et Lille. Les deux premières offrent des diplômes uniquement reconnus dans leur propre cadre tandis que Strasbourg délivre des diplômes d'État (DESS en traduction et en interprétation de conférence) et que l'école de Lille jouit d'un statut analogue à celui de l'ISIT.

Grande-Bretagne: Les formations britanniques sont très diverses (voir aussi note 4). Elles sont essentiellement universitaires et entrent dans le cadre de départements, facultés ou écoles de langues modernes. Seules les formations de Colchester et de Warwick s'inscrivent dans des départements de littérature ou de littérature comparée. Ce sont des formations plutôt orientées vers les théories

2. Les instituts catholiques constituent en France une catégorie particulière au sein du système de l'enseignement supérieur à côté des universités, des grandes écoles et des instituts d'études politiques. Ce sont des établissements privés reconnus par le ministère de l'Éducation nationale. 
relatives à la problématique de l'interculturalité ${ }^{3}$. Un autre cas particulier est celui de l'UMIST (University of Manchester Institute of Science and Technology) qui offre un cursus en traduction automatique. En règle générale, les formations en traduction et interprétation sont assez nombreuses en Grande-Bretagne, mais il n'existe pas dans ce pays de structures propres pour ces disciplines.

Grèce: Il existe à notre connaissance une seule formation en traduction et interprétation en Grèce: au sein du Département de langues étrangères, traduction et interprétation de l'Université de Ionie (Corfou); elle débouche sur un double diplôme en traduction et en interprétation équivalant à la maîtrise française.

Irlande: Les formations irlandaises s'apparentent, du point de vue de leur statut, aux formations anglaises. Les deux institutions recensées (University College de Cork et Dublin City University) offrent des cursus universitaires de traduction et d'interprétation dans le cadre d'écoles de langues ou de langues appliquées et débouchent sur des B.A. ou M.A.

Italie: À l'heure actuelle, deux universités seulement - Bologne et Trieste - offrent des cursus en traduction et interprétation. Pour le reste, la formation des traducteurs et interprètes est principalement le fait des Scuoli Superiori per Interpreti e Traduttori non universitaires qui proposent des formations professionnelles de trois ans. Cette situation semble destinée à changer, les formations devant à l'avenir faire l'objet de formations universitaires de type long (laurea). On peut remarquer qu'en Italie, comme en Belgique, les formations en traduction et interprétation s'intègrent dans des structures propres (Scuoli Superiori per Interpreti e Traduttori), même au sein des deux universités (Scuoli Superiori di Lingue Moderne per Interpreti e Traduttori).

3. Comme aussi, ailleurs en Europe, à Paris X (Nanterre), à Leuven (Belgique), etc. 
Luxembourg: Il n'y a aucune formation en traduction ou interprétation au Luxembourg au niveau de l'enseignement supérieur.

Norvège: On ne trouve qu'une seule formation de traducteurs en Norvège. C'est le Agder College de Kristiansand - établissement d'enseignement supérieur non universitaire - qui offre une formation professionnelle de traducteur technique de trois ans. À signaler aussi que l'école de commerce de Bergen organise des examens officiels pour les traducteurs assermentés (statsautorisert translator) et qu'elle est en train de mettre en place une formation spécifique pour cet examen.

Pays-Bas: Il n'existe plus au Pays-Bas qu'une formation universitaire en traduction et interprétation: à Groningue (l'Institut d'Amsterdam ayant dû fermer), à laquelle on peut ajouter la formation non universitaire de Maastricht (pour un diplôme de traducteur professionnel de quatre ans à partir de l'accès à l'enseignement supérieur). La formation universitaire ${ }^{4}$ conduit à un Doctorandus vertaalwetenschap.

Portugal: Le Portugal offre quelques similitudes avec l'Italie dans la mesure où la plupart des formations sur trois ou quatre ans relève des Institutos Superiores de Linguas e Administração non universitaires. Au niveau des universités, on trouve uniquement des formations courtes de moyen ou haut niveau (Diplomas de especializção). Il ne semble pas qu'il y ait à l'heure actuelle au Portugal de réforme en cours concernant ces formations.

4. On peut ajouter deux autres formations universitaires en ce qui concerne les Pays-Bas: l'une est offerte par l'Université de Nimègue et l'autre par l'Université d'Utrecht. Mais ces deux formations ne débouchent pas sur un diplôme spécifique en traduction ou en interprétation. Elles constituent une spécialisation au sein de cursus plus généraux en langues étrangères. 
Suède: Le cas de la Suède est peut-être le plus particulier d'entre tous. Il existe une seule institution consacrée à la formation des traducteurs et interprètes: l'Institut d'études en interprétation et en traduction de l'Université de Stockholm (Tolk-och översättarinstitutet vid Stockholms universitet). Cet institut fonctionne comme une sorte de "centre conseil» pour la formation des traducteurs et interprètes. Sur la base d'un budget alloué par le gouvernement suédois, il définit, organise et met en œuvre - en coordination avec les différentes écoles ou départements universitaires de langue - des programmes de formation très variés: formation de traducteurs, d'interprètes communautaires, organisée au niveau universitaire, formation d'interprètes de liaison et d'interprètes pour malentendants au niveau des formations professionnelles et des formations continues pour adultes. Seule la formation pour les interprètes de conférence est directement assurée par l'Institut.

Suisse: La formation des traducteurs et interprètes en Suisse est principalement représentée par l'École de Traduction et d'Interprétation de l'Université de Genève, l'une des plus anciennes en Europe puisqu'elle a été créée en 1941. Il existe aussi une institution non universitaire privée, l'École d'Interprètes de Zurich, qui offre une double formation professionnelle de traducteurs et d'interprètes.

Il n'y a donc pas deux pays en Europe de l'Ouest qui présentent la même situation. Au-delà des différences notables entre les systèmes éducatifs nationaux, la façon dont les disciplines de la traduction et de l'interprétation s'intègrent dans l'enseignement supérieur rend très difficile de les situer. Mais cette difficulté est peut-être inhérente aux disciplines elles-mêmes. La pratique de la traduction et de l'interprétation s'applique en effet à des domaines multiples. Leur enseignement peut alors se trouver, tout comme l'enseignement des langues étrangères, dans des situations institutionnelles tout à fait diverses. Tantôt la traduction et l'interprétation, qui servent le commerce et l'industrie, sont des formations dans des écoles de commerce. Tantôt, au service aussi des échanges culturels, littéraires et cinématographiques, elles 
prennent place dans des départements universitaires de littérature ou de langues appliquées. Tantôt enfin, pour aider également aux échanges massifs d'informations à l'échelle des nations ou des institutions européennes, notamment grâce aux moyens informatisés, elles se développent dans des instituts de technologie.

La diversité des structures institutionnelles et le statut qu'on accorde à la traduction au sein de l'enseignement supérieur ne posent pas problème en soi. Ils posent problème à partir du moment où l'on veut faire de la traduction un domaine propre, où l'on cherche à définir sa spécificité par rapport aux autres domaines. Cette question est bien réelle - le nombre des formations qui se sont créées dans les années 80-90 et les réformes concernant ces formations dans divers pays le démontrent. Mais elle est beaucoup plus liée à l'intégration progressive des formations dans le cadre universitaire et à la place qu'elles doivent y prendre qu'à des révélations purement théoriques sur la traduction.

\section{Le profil des formations (durée et niveau d'études)}

Le profil des formations relève de la même problématique. La place que la traduction doit occuper dans l'université dépend aussi de sa capacité à justifier de formations de type universitaire. On note alors une évolution dans l'abandon progressif des formations moyennes - autrefois dispensées dans des écoles ou instituts non universitaires - au profit des formations longues (entre quatre et cinq années d'études) et des formations courtes (après trois ou quatre années d'études) de moyen ou haut niveau.

Ces formations, aujourd'hui dominantes, représentent respectivement $39 \%$ et $41,5 \%$, soit environ $80 \%$ du total des formations. Les formations moyennes (trois ans à partir de l'accès à l'enseignement supérieur) représentent quant à elles $20 \%$ environ de ce total.

Les formations longues sont surtout représentées en Belgique, en Espagne, en Allemagne, en Autriche, en Finlande, au Danemark ou aux Pays-bas. Les formations courtes de moyen ou 


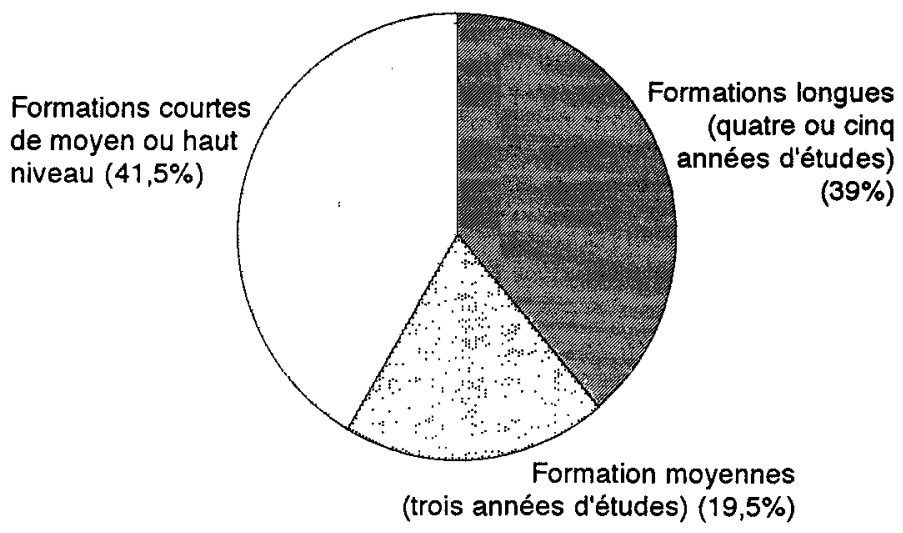

haut niveau sont particulièrement fréquentes en France et en Grande-Bretagne. En Belgique, en Espagne ou au Portugal, on voit aussi se développer, parallèlement aux formations longues, ce type de formation. Les formations moyennes se trouvent principalement au Portugal et en Italie. Il semble toutefois que les formations italiennes soient destinées à être rattachées au cadre universitaire. Elles suivraient alors la même évolution que les diplomaturas espagnoles de trois ans délivrées autrefois par les Escuelas universitarias puis intégrées, par voie de réforme, aux formations universitaires longues (licenciaturas).

La différence des formations longues et des formations courtes de moyen ou haut niveau s'explique en partie par la différence des systèmes éducatifs nationaux. Mais elle s'explique aussi par la place accordée au perfectionnement des langues et aux domaines de spécialité au sein des formations données. Les filières longues intègrent habituellement l'enseignement des langues et l'initiation à un domaine de spécialité. Bref, les plans d'études se divisent en quatre grands blocs de matières constitués de façon plus ou moins égale en terme d'heures de cours: les matières relatives à 
l'enseignement des langues, à un ou plusieurs domaines de spécialité - «matières thématiques» ou "matières d'application» -, aux domaines afférents à la traduction - «matières instrumentales» (terminologie, informatique appliquée à la traduction, etc.) - et à la pratique et théorie de la traduction et de l'interprétation. Les formations courtes, en revanche, misent sur les acquis préalables des étudiants tant en ce qui concerne la connaissance des langues étrangères que le degré de spécialisation dans un domaine donné. L'enseignement peut alors se centrer sur la pratique de la traduction et de l'interprétation et sur les matières instrumentales.

Ces deux modèles - formations longues et formations courtes de moyen ou haut niveau - représentent en réalité deux options différentes au sein de la formation des traducteurs et interprètes. Le choix entre ces options dépend bien plus souvent de facteurs tels que la structure des systèmes éducatifs nationaux, le niveau et le développement de l'enseignement des langues dans les différents pays, les stratégies choisies pour introduire les disciplines de la traduction et de l'interprétation dans le domaine universitaire, etc., que de considérations théoriques sur un modèle idéal de formation.

Il existe enfin en Europe une dizaine de formations doctorales en traduction. La traduction se situerait donc à mi-chemin entre une activité professionnelle qui suppose une préparation technique et pratique assurée par des professionnels et un domaine d'études et de recherches qui suppose une préparation théorique assurée par des universitaires. Si la séparation des deux orientations est assez claire d'un point de vue conceptuel - d'un côté la pratique et de l'autre la théorie -, elle n'est pas aussi claire au niveau des formations universitaires qui poursuivent aussi un but scientifique.

Deux orientations semblent se dessiner sur la façon d'introduire la traduction dans les domaines d'études et de recherches universitaires. En témoignent les deux formations doctorales de Paris (ESIT) et de Warwick en Grande-Bretagne. La première s'intitule Doctorat en traductologie et la deuxième $P h D$ in Translation Studies, Comparative Literary Theory, Comparative 
Literature, British Cultural Studies, Modern British Studies and Post-Colonial Studies. Dans le premier cas, la traduction est envisagée comme une science ayant pour objet propre l'étude des processus mis en place pour effectuer l'opération interlinguistique. Dans le deuxième cas, la traduction est envisagée sous l'angle de l'interdisciplinarité avec les théories littéraires et sociologiques. La traduction peut-elle ou doit-elle se constituer comme une discipline à part entière ou comme une «interdiscipline» qui embrasserait des domaines aussi divers que la linguistique, l'histoire, la sociologie, la psychologie, la théologie, voire les mathématiques, l'informatique, etc.?

\section{Le rapport traduction/interprétation}

On observe, sur l'ensemble des formations, quatre modèles différents du rapport traduction - interprétation: le modèle $\mathbf{Y}$ où la traduction et l'interprétation font l'objet de deux formations spécialisées après un cursus d'études communes; le modèle global où la traduction et l'interprétation font l'objet d'une formation commune et où tous les étudiants sont formés à la fois à la traduction et à l'interprétation; le modèle progressif où la formation en interprétation intervient après un cursus d'études en traduction; le modèle parallèle où la traduction et l'interprétation font l'objet de deux cursus parallèles, au sein de la même institution.

Le schéma suivant représente la proportion de chacun de ces modèles sur l'ensemble des formations recensées. Le modèle $\mathrm{Y}$ et le modèle global dominent largement l'ensemble des formations. Le modèle $\mathbf{Y}$ est surtout représenté en Allemagne, Autriche, Belgique, Italie et on le retrouve aussi en Grèce. Ces pays présentent en général des formations communes de une, deux ou trois années selon les cas, débouchant sur deux formations séparées en traduction et en interprétation. Ce modèle peut parfois se combiner avec le modèle progressif. Certaines formations belges en effet offrent aux licenciés-traducteurs la possibilité de se spécialiser en interprétation en accomplissant une cinquième année d'études. Cette procédure, de plus en plus fréquente, se concrétise dans certaines cas par la mise 


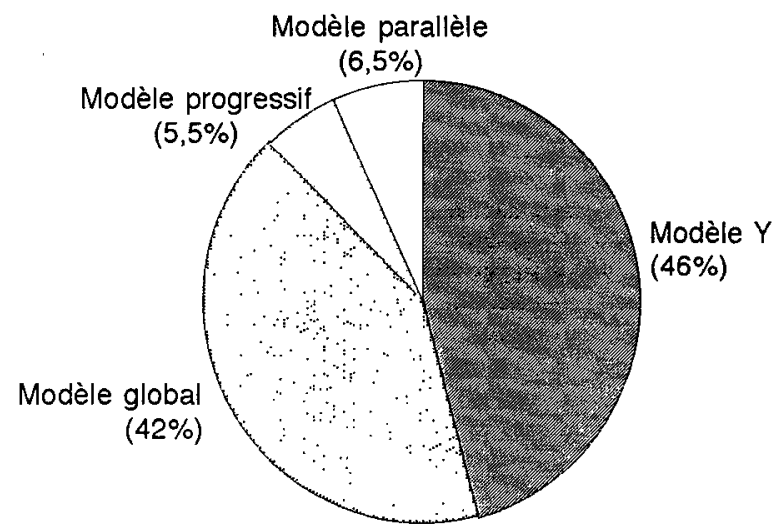

en place d'un postgraduat en interprétation de conférence auquel sont admis les étudiants ayant achevé leurs études en traduction (Katholieke Vlaamse Hogeschool, Anvers; Institut Libre Marie Haps, Bruxelles). Ces institutions présentent donc dans un premier temps un cursus commun aboutissant à une double spécialisation en traduction ou en interprétation puis, dans un deuxième temps, un cursus d'interprétation ouvert aux étudiants préalablement formés à la traduction.

Le modèle global est principalement représenté en Espagne, au Danemark, en Finlande. On le trouve aussi, quoique de façon très accidentelle en France et en Grande-Bretagne ${ }^{5}$. L'Université de

5. La seule institution en France à offrir ce type de cursus est l'Ecole Supérieure de Cadres Interprètes-Traducteurs de l'Université de Toulouse/Le Mirail. En Grande-Bretagne, la plupart des institutions se consacrent à la traduction. Quatre institutions offrent à notre connaissance des cursus en traduction et interprétation: School of Modern Languages, University of Bath; Heriot-Watt University, Edimbourg; School of Languages, University of Westminster; Department of Modern Languages, University of 
Dublin en Irlande offre aussi un $B A$ in Applied Languages (Translation with Interpretation) conçu selon ce modèle.

La principale caractéristique du modèle global est que l'interprétation y fait seulement l'objet d'une initiation. Le plan d'études de la licenciatura espagnole propose au niveau ministériel une formation globale en traduction et interprétation. L'enseignement de l'interprétation représente une part infime des unités de valeur dans le cadre de ce plan (16 sur 106 au total). Étant donné que $30 \%$ seulement du total de la formation est définie par ce plan et que les universités créent sur cette base leur propre plan d'études $(60 \%$ des unités de valeur sont déterminées par les universités et les $10 \%$ restant sont librement choisies par les étudiants), chaque institution peut proposer, si elle le désire, un enseignement plus approfondi de l'interprétation. Dans l'immense majorité des cas, cependant, le manque d'interprètes professionnels disponibles et l'absence d'installations font que ces formations sont essentiellement orientées vers la traduction.

En ce qui concerne le Danemark, la Finlande, 1'Université de Dublin en Irlande et quelques institutions anglaises, le modèle global en traduction et interprétation relève, semble-t-il, d'une conception plus générale des métiers de la traduction. Il s'agit dans la plupart des cas de former non pas des traducteurs ou des interprètes mais des professionnels de la communication interculturelle qui seront amenés, dans le cadre d'activités relatives principalement au commerce ou à l'industrie, à accomplir toutes sortes de tâches orales et écrites. L'accent ne porte donc pas tant sur les activités (traduction ou interprétation) que sur la communication interculturelle en général, laquelle englobe tout un ensemble d'activités (traduction, rédaction, interprétation, etc.). Dans ce type

Salford. Nous disposons des plans d'études uniquement pour les deux premières. La formation d'Edimbourg est conçue selon le modèle global. Celle de l'Université de Westminster est conçue selon le modèle parallèle: la traduction et l'interprétation font l'objet de deux formations séparées. 
de cursus, l'interprétation fait alors l'objet d'une spécialisation, obligatoire ou optionnelle selon les cas, mais toujours minime, au sein de la formation.

Il faut noter cependant que l'interprétation de conférence n'entre pas ou très peu dans le cadre de ces formations. L'Espagne ou la Grande-Bretagne proposent, en plus des cursus universitaires globaux, des formations courtes de haut niveau en interprétation de conférence ${ }^{6}$. Copenhague, Stockholm et Turku (Finlande) proposent aussi une formation spécifique en ce domaine, en particulier pour répondre aux besoins des institutions de l'Union Européenne.

Enfin, une dernière remarque doit être faite à propos de certaines formations globales que l'on trouve en France, en Italie ou au Portugal. En France, l'École Supérieure de Cadres Interprètes-Traducteurs de Toulouse propose un cursus de deux ans à partir du DEUG (2 années d'études). En Italie, la Scuola Superiore per Interpreti e Traduttori de Milan, qui dispose de diverses annexes (Rome, Naples, Florence, Bologne, Bari et Gênes), offre un Diploma di Traduttore ed Interprete Corrispondente in Lingue Estere de deux ans à partir de l'accès à l'enseignement supérieur. Au Portugal, l'Instituto Superior de Linguas e Administração (Porto) dispense une formation en traduction et interprétation de trois ans à partir de l'accès à l'enseignement supérieur. Ces formations préparent les étudiants à des professions de secrétariat et de relations publiques dans le cadre du commerce et de l'entreprise ou, originalement en ce qui concerne Milan, des ambassades ou des ministères de l'économie ou des affaires étrangères.

La différence entre ces formations et les formations danóises ou finlandaises réside en premier lieu dans la durée et le niveau d'études. Elle réside aussi dans la conception de la finalité

6. En Espagne: Universitat Autònoma de Barcelona; Universidad de La Laguna. En Grande-Bretagne: University of Westminster-Euston Centre. 
professionnelle des formations. Dans un cas (Danemark, Finlande), l'approche théorique des problèmes de la communication interculturelle implique une plus grande responsabilité de la part du professionnel dans la réalisation de cette communication. Dans le cas des écoles française, italienne ou portugaise, la formation reste essentiellement centrée sur les activités de traduction et d'interprétation.

Les deux derniers modèles sont tout à fait minoritaires. Le modèle progressif est représenté, outre les formations belges mentionnées plus haut, par deux autres institutions: l'Institut de Perfectionnement en Langues Vivantes d'Angers et l'École de Traducteurs et d'Interprètes de Genève. Le modèle parallèle est représenté en France (ESIT, ISIT et Strasbourg), en Grande-Bretagne (University of Westminster, Londres) et en Italie (SSIT «San Pellegrino», Misano Adriatico). Si dans le cadre de certains cursus, on trouve quelques matières communes aux deux formations, elles sont tout à fait minimes et ne sauraient constituer, comme dans le modèle $\mathrm{Y}$, un tronc commun de formation.

\section{Conclusion}

La diversité inextricable du statut des formations en traduction et interprétation dans l'enseignement supérieur de chaque pays découle de plusieurs facteurs. Tout d'abord, ces formations sont relativement récentes dans le cadre de l'enseignement supérieur - qui est lui-même en constante réforme - et n'ont pas encore su déterminer leur visée profonde (en gros entre formation professionnelle et formation universitaire) et la place qu'elles peuvent revendiquer au sein de cet enseignement. Ensuite, ces formations ont bénéficié jusqu'ici d'une attention et d'un soutien variables de la part des ministères de l'Éducation. Les programmes d'études se créent souvent sur des initiatives particulières dans le cadre d'une conjoncture économique favorable. Dans divers pays, notamment en Allemagne et aux Pays-Bas, certaines formations sont menacées pour des raisons budgétaires. La traduction et l'interprétation constituent 
donc au sein de l'enseignement supérieur un domaine relativement jeune, mal défini et instable.

On devine cependant, en ce qui concerne le profil des formations et le rapport traduction/interprétation au sein de ces formations, quelques tendances: les cursus d'études longs rivalisent avec les cursus courts de moyen ou haut niveau dans la formation des professionnels tandis que les cursus moyens tendent à disparaître. La coexistence des deux types (longs et courts) constitue à notre avis un élément fondamental de structuration pour les deux disciplines concernées dans le cadre universitaire. Les formations universitaires longues peuvent en effet déboucher aussi bien sur la recherche (formations doctorales) ou sur l'enseignement que sur la vie professionnelle tandis que les formations courtes de haut niveau sont exclusivement consacrées à la formation professionnelle. Ces formations remplissent ainsi dans leur ensemble un double objectif professionnel et universitaire qui leur permet de se renouveler au sein de l'université.

En ce qui concerne le rapport traduction/interprétation, les grandes tendances se cristallisent autour des modèles $\mathrm{Y}$ et global. Comme nous l'avons dit plus haut, ces deux modèles constituent un véritable axe de réflexion sur le devenir des formations en traduction et interprétation: formations de traducteurs, d'interprètes ou plus généralement de professionnels de la communication interculturelle. Il est à noter que certains pays où le modèle $\mathrm{Y}$ est dominant notamment Allemagne, Autriche, Belgique et Italie - sont des pays où les premières formations en traduction et interprétation sont apparues dès les années 40-50.

S'il fallait un cadre de concertation pour réduire ces diversités ou du moins y introduire une plus grande cohérence, ce ne serait certainement pas le cadre national. Les recherches que nous avons faites pour établir le recensement des formations sur lequel nous nous sommes appuyée ont montré que l'espace de communication et de coopération entre les institutions se situait bien plus au niveau international - c'est à dire au niveau européen - 
qu'au niveau national ${ }^{7}$. La concertation entre les institutions d'un même pays reste en général très limitée. Les échanges, les rencontres et les réseaux qui se constituent et qui sont les véritables foyers de réflexion se situent au-delà des frontières nationales. Peut-être alors faut-il penser que la seule voie d'avenir pour les disciplines de la traduction et de l'interprétation, ou plus exactement de la communication interculturelle, réside d'abord dans la définition de leur visée profonde au niveau de l'Europe, de l'Ouest et de l'Est.

Monique Caminade: 44610 Calaceite (Teruel) Espagne

\section{RÉSUMÉ: Les formations en traduction et interprétation:} Perspectives en Europe de l'Ouest - Cet article propose une analyse globale des programmes de formation et interprétation dans les pays d'Europe de l'Ouest. L'analyse s'effectue autour de trois axes 1) le statut de ces formations dans le cadre institutionnel de l'enseignement supérieur des différents pays; 2) le profil des formations (courtes, moyennes, longues); 3) le rapport des disciplines de la traduction et de l'interprétation au sein de ces

7. Notre première démarche a été de demander aux différentes institutions une liste des institutions offrant des cursus en traduction et en interprétation dans leur propre pays. Cette démarche s'est révélée très peu fructueuse: manque de volonté, manque de temps, mais aussi absence réelle parfois d'information sur les institutions nationales. Nous avons alors demandé aux différentes institutions une liste des institutions avec lesquelles elles étaient en relation de coopération, grâce par exemple à CIUTI (Conférence internationale des Instituts Universitaires de Traducteurs et Interprètes), à EST (European Society for Translation Studies), aux programmes d'échanges d'étudiants et d'enseignants comme Erasmus, etc. C'est par ce biais, c'est-à-dire par les multiples recoupements que nous avons pu établir entre diverses listes internationales - le plus souvent européennes que nous avons constitué notre annuaire. 
formations. Cette analyse a été faite sur la base d'un recensement de 136 institutions dans dix-sept pays au cours de l'année 1994.

\begin{abstract}
Translators' and Interpreters' Training: Perspectives in Western Europe - West-European programmes for the training of translators and interpreters are analysed on three levels: 1) the status of these programmes within higher education institutions in the various countries, 2) their nature and duration (short, medium, long), and 3) their interdisciplinary make-up with respect to translation and/or interpretation. The corpus for the study, compiled in 1994, comprises 136 institutions in seventeen countries.
\end{abstract}

